CUBO A Mathematical Journal Vol.13, No-02, (119-126). June 2011

\title{
Degree theory for the sum of VMO maps and maximal monotone maps
}

\author{
YUQING CHEN \\ Faculty of Applied Mathematics, \\ Guangdong University of Technology, \\ Guangdong 510006, P. R. China, \\ email: ychen640163.com \\ DONAL O'REGAN \\ Department of Mathematics, \\ National University of Ireland, \\ Galway, Ireland, \\ email: donal.oregan@nuigalway.ie \\ and \\ Ravi P. Agarwal \\ Department of Mathematical Science, \\ Florida Institute of Technology \\ Melbourne, FL, 32901, USA, \\ email: agarwal@fit.edu
}

\begin{abstract}
Let $\Omega \subset \mathrm{R}^{\mathrm{n}}$ be an open bounded domain, $f: \Omega \rightarrow \mathrm{R}^{\mathrm{n}}$ a VMO map, and $\mathrm{T}: \mathrm{D}(\mathrm{T}) \subseteq$ $R^{n} \rightarrow R^{n}$ a maximal monotone map with $\mathrm{D}(\mathrm{T}) \cap \Omega \neq \emptyset$. We construct a degree for the sum of $f+T$, which can be viewed as a generalization of the degree both for VMO maps and maximal monotone maps.
\end{abstract}




\section{RESUMEN}

Sea $\Omega \subset R^{n}$ un dominio abierto, $f: \Omega \rightarrow R^{n}$ un mapa VMO, y $T: D(T) \subseteq R^{n} \rightarrow R^{n}$ un mapa monotono maximal con $\mathrm{D}(\mathrm{T}) \cap \Omega \neq \emptyset$. Construimos un grado por la suma de $\mathrm{f}+\mathrm{T}$, que se puede ver como una generalización de la medida, tanto para los mapas de VMO y para los mapas monotono maximal.

Keywords and phrases: Degree theory, Maximal monotone map. Mathematics Subject Classification: 47H11, 47H05

\section{Introduction}

Degree theory for continuous maps in finite dimensional spaces has a long history and has been extensively studied. In the early 80's of the last century a degree for some classes of noncontinuous maps was established (see $[8,1,17,18]$ and the references therein). In 1995 and 1996, H. Brezis and L. Nirenberg [12], [13] invented a degree theory for VMO maps; see [2-6,9-11,19,21,22]. Generally, VMO functions need not be continuous. Another important class of non-continuous maps is the class of maximal monotone maps, and there is no relation between the VMO maps and the maximal monotone maps. In this paper, we consider the sum of a VMO map and a maximal monotone map, and we will define a degree theory for such a map. First we recall some definitions. Let $\Omega$ be an open bounded domain in $\mathrm{R}^{\mathrm{n}}$. The class of bounded mean oscillation functions (see [20]) are defined as

$$
\mathrm{BMO}(\Omega)=\left\{\mathrm{f}: \Omega \rightarrow \mathrm{R}^{\mathrm{n}} \text { is locally integrable, and }|\mathrm{f}|_{\mathrm{BMO}}<\infty\right\}
$$

where $|f|_{B M O}=\sup _{B \subset \Omega} \frac{1}{m(B)} \int_{B}|f(x)-\bar{f}| d x, \bar{f}=\frac{1}{m(B)} \int_{B} f(x) d x$ (here $m(\cdot)$ represents the Lebesgue measure), and the class of vanishing mean oscillation functions (see [23]) are defined as

$$
\operatorname{VMO}(\Omega)=\left\{f: \Omega \rightarrow R^{n} \text { is locally integrable, and } \lim _{m(B) \rightarrow 0} \frac{1}{m(B)} \int_{B}|f(x)-\bar{f}| d x=0\right\},
$$

where $B \subset R^{n}$ is an open ball with its closure contained in $\Omega$. It is well known that if $f \in \mathrm{VMO}$, then $f_{\epsilon}(x)=\frac{1}{m\left(B_{\epsilon}(x)\right)} \int_{B_{\epsilon}(x)} f(y) d y$ is continuous in $\epsilon$ and $x$ where it is defined. Let $T: D(T) \subset$ $R^{n} \rightarrow R^{n}$ be a function. If $(h-g, x-y) \geq 0$ for all $x, y \in D(T)$ and $h \in T x, g \in T y$, then $T$ is said to be monotone. If $T$ is monotone and $T$ has no monotone extension in $R^{n}$, then $T$ is said to be maximal monotone. It is well known that $T$ is maximal monotone iff $T$ is monotone and $T+\epsilon I$ is surjective for all $\epsilon>0$. If $T$ is maximal monotone, we use $T_{\epsilon}=\left(T^{-1}+\epsilon I\right)^{-1}$ to represent the Yosida approximation, and $R_{\epsilon}=I-\epsilon T_{\epsilon}$, the resolvent with respect to $T_{\epsilon}$. For maximal monotone maps we refer the reader to [7]. Let $f: \Omega \rightarrow R^{n}$ be a VMO map, $T: D(T) \subseteq R^{n} \rightarrow R^{n}$ a maximal monotone map, $p \in R^{n}$, and $\mathrm{D}(\mathrm{T}) \cap \Omega \neq \emptyset$. Under appropriate assumptions, see (2.1) below, we define the degree $\operatorname{deg}(f+T, \Omega \cap D(T), p)$. If $T=0$, this degree coincides with the degree for $\mathrm{VMO}$ 
maps in [13], and if $f=0$, then it coincides with the degree for maximal monotone maps (see $[14-16])$.

\section{Results}

In this section, $\Omega \subset \mathrm{R}^{\mathrm{n}}$ is an bounded open domain, $f \in \operatorname{VMO}(\Omega), \mathrm{T}: \mathrm{D}(\mathrm{T}) \subseteq \mathrm{R}^{\mathrm{n}} \rightarrow \mathrm{R}^{\mathrm{n}}$ is a maximal monotone map, $p \in R^{n}$, and $\Omega \cap D(T) \neq \emptyset$. Suppose there exists an open neighborhood $\mathrm{U}$ of $\partial \Omega$ in $\Omega$ and a constant $\beta>0$ such that

$$
\frac{1}{m\left(B_{\epsilon}(y)\right)} \int_{B_{\epsilon}(y)}|f(x)+g-p| d x \geq \beta
$$

for all $0<\epsilon<\frac{1}{2} \mathrm{~d}(\mathrm{y}, \partial \Omega), \mathrm{g} \in \mathrm{T} z, z \in \mathrm{D}(\mathrm{T}) \cap \mathrm{B}_{\epsilon}(\mathrm{y})$, where $\mathrm{B}_{\epsilon}(\mathrm{y})$ is an open ball centered at $\mathrm{y}$ with radius $\epsilon$ such that $\overline{\mathrm{B}_{\epsilon}(\mathrm{y})} \subset \mathrm{U}$, and $\mathrm{d}(\mathrm{y}, \partial \Omega)$ is the distance between $\mathrm{y}$ and $\partial \Omega$.

We remark that if $T=0$, then (2.1) was first used in [13]. If $f=0$, then (2.1) is equivalent to $|g-p| \geq \beta$ for all $z \in \mathrm{D}(\mathrm{T}) \cap \mathrm{U}$ and $\mathrm{g} \in \mathrm{T} z$, and in this case Proposition 2.1 below shows that the assumption $p \notin \mathrm{T}(\partial \Omega \cap \mathrm{D}(\mathrm{T}))$ will guarantee (2.1) holds.

Proposition 2.1. If $p \notin T(\partial \Omega \cap D(T))$, then there exists $d_{0}>0, \alpha_{0}>0$ such that $d(p, T x) \geq$ $\mathrm{d}_{0}$ for all $x \in \Omega \cap \mathrm{D}(\mathrm{T})$ with $\mathrm{d}(x, \partial \Omega)<\alpha_{0}$.

Proof. Suppose the conclusion is not true. There exist $x_{n} \in \Omega \cap D(T), g_{n} \in T x_{n}$ such that $\mathrm{d}\left(\mathrm{x}_{\mathrm{n}}, \partial \Omega\right) \rightarrow 0$, and $\mathrm{g}_{\mathrm{n}}-\mathrm{p} \rightarrow 0$. Without loss of generality, we may assume that $x_{n} \rightarrow x_{0} \in \partial \Omega$.

Since $\left(g_{n}-g, x_{n}-x\right) \geq 0$ for all $x \in D(T), g \in T x$, we have

$$
\left(p-g, x_{0}-x\right) \geq 0, \text { for all } x \in D(T), g \in T x
$$

Therefore $x_{0} \in \partial \Omega \cap D(T), p \in T x_{0}$, which is a contradiction.

As in [13], we define $\Omega_{\epsilon}=\{x \in \Omega: d(x, \partial \Omega)>2 \epsilon\}$ for each $\epsilon>0$. By definition of VMO functions, there exists $\epsilon_{0}>0$ such that

$$
\frac{1}{m\left(B_{\epsilon}(x)\right)} \int_{B_{\epsilon}(x)}|f(y)-\bar{f}| d y<\frac{\beta}{2}
$$

for all $\epsilon<\epsilon_{0}, x \in \Omega$ and $\epsilon<\frac{d(x, \partial \Omega)}{2}$. We may also take $\epsilon_{0}$ such that $\left\{x \in \Omega: d(x, \partial \Omega) \leq 3 \epsilon_{0}\right\} \subset \mathrm{U}$, where $\mathrm{U}$ is the same as in (2.1). Now for $0<\epsilon<\epsilon_{0}$, and $x \in \partial \Omega_{\epsilon} \cap \mathrm{D}(\mathrm{T}), \mathrm{g} \in \mathrm{Tx}$, by (2.1) and (2.2), we obtain

$$
\left|f_{\epsilon}(x)+g-p\right| \geq \frac{\beta}{2},
$$

where $f_{\epsilon}(x)=\frac{1}{m\left(B_{\epsilon}(x)\right)} \int_{\left.B_{\epsilon}(x)\right)} f(y) d y$. 
Lemma 2.2. Suppose $\left|f_{\epsilon}(x)+g-p\right| \geq \frac{\beta}{2}$, for $x \in \partial \Omega_{\epsilon} \cap D(T), g \in T x$. Then there exists $\lambda_{0}(\epsilon)>0$ such that

$$
p \neq f_{\epsilon}(x)+T_{\lambda}(x), \text { for all } x \in \partial \Omega_{\epsilon}, \lambda \in\left(0, \lambda_{0}(\epsilon)\right) \text {. }
$$

Proof. If this is not true, there exist $\lambda_{n} \rightarrow 0^{+}, x_{n} \in \partial \Omega_{\epsilon}$ with $x_{n} \rightarrow x_{0} \in \partial \Omega_{\epsilon}$, such that

$$
f_{\epsilon} x_{n}+T_{\lambda_{n}} x_{n}=p, \quad n \in\{1,2, \cdots\}
$$

Since $f_{\epsilon} x_{n} \rightarrow f_{\epsilon} x_{0}, R_{\lambda_{n}} x_{n}=x_{n}-\lambda_{n} T_{\lambda_{n}} x_{n} \rightarrow x_{0}$, the maximal monotonicity of $T$ implies that $x_{0} \in D(T)$, and $p-f_{\epsilon} x_{0} \in T x_{0}$, which is a contradiction.

Now, assume that (2.1) holds. In view of (2.3) and Lemma 2.2, we define the $\operatorname{degree} \operatorname{deg}(\mathrm{f}+$ $\mathrm{T}, \Omega \cap \mathrm{D}(\mathrm{T}), \mathrm{p})$ by

$$
\operatorname{deg}(f+T, \Omega \cap D(T), p)=\lim _{\epsilon \rightarrow 0^{+}} \lim _{\lambda \rightarrow 0^{+}} \operatorname{deg}\left(f_{\epsilon}+T_{\lambda}, \Omega_{\epsilon}, p\right) .
$$

We claim this definition is reasonable. First, for each $\epsilon<\epsilon_{0}$, and $\lambda_{1}, \lambda_{2} \in\left(0, \lambda_{0}(\epsilon)\right)$, since $T_{t} \lambda_{1}+(1-t) \lambda_{2} x$ is continuous in $(t, x)$ (see Corollary 2.8 in [15]) we know that $\left\{f_{e}+T_{t \lambda_{1}+(1-t) \lambda_{2}}\right\}_{t \in[0,1]}$ is a homotopy, so

$$
\operatorname{deg}\left(f_{\epsilon}+T_{\lambda_{1}}, \Omega_{\epsilon}, p\right)=\operatorname{deg}\left(f_{\epsilon}+T_{\lambda_{2}}, \Omega_{\epsilon}, p\right) .
$$

Now, for any $\epsilon \in\left(0, \epsilon_{0}\right)$, by the continuity of $f_{t}(x)$ in $(t, x)$ and $(2.3)$, there exists $\delta>0$ such that

$$
\left|f_{t}(x)+g-p\right|>\frac{\beta}{4}
$$

for $|\mathrm{t}-\epsilon| \leq \delta$ and $x \in \partial \Omega_{\epsilon}$ and $\mathrm{g} \in \mathrm{T} x$. The same proof as in Lemma 2.2 guarantees that there exists $\lambda_{1}>0$ such that

$$
p \neq f_{t}(x)+T_{\lambda}(x) \text {, for all } x \in \partial \Omega_{\epsilon},|t-\epsilon| \leq \delta, \lambda \in\left(0, \lambda_{1}\right) \text {, }
$$

so $\operatorname{deg}\left(f_{t}+T_{\lambda}, \Omega_{\epsilon}, p\right)$ is well defined for $\lambda \in\left(0, \lambda_{1}\right)$, and $|t-\epsilon| \leq \delta$. By homotopy invariance, we have

$$
\operatorname{deg}\left(f_{t}+T_{\lambda}, \Omega_{\epsilon}, p\right)=\operatorname{deg}\left(f_{\epsilon}+T_{\lambda}, \Omega_{\epsilon}, p\right),
$$

so the degree in (2.4) is well defined.

For a measurable function $f: \Omega \rightarrow R^{n}$, we recall that the essential range of $f$ is defined as the smallest closed subset essR(f) such that $f(x) \in \operatorname{essR}(f)$ a. e. $x \in \Omega$ (see [12]).

Proposition 2.3. If $\operatorname{deg}(f+T, \Omega \cap D(T), p) \neq 0$, then $p \in \overline{\operatorname{essR}(f)+T(\bar{\Omega} \cap D(T)}$.

Proof. Suppose the conclusion is not true. Then exists $r>0$ such that $B(p, r) \cap \operatorname{essR}(f)+$ $\mathrm{T}(\bar{\Omega} \cap \mathrm{D}(\mathrm{T}))=\emptyset$. Set $\Sigma=\mathrm{R}^{n} \backslash(\mathrm{B}(\mathrm{p}, \mathrm{r})-\mathrm{T}(\bar{\Omega} \cap \mathrm{D}(\mathrm{T})))$. Clearly, essR $(\mathrm{f}) \subset \Sigma$. Also $f(x) \in \operatorname{ess} \mathrm{R}(\mathrm{f})$, 
a. e. $x \in \Omega$, and $f \in \operatorname{VMO}(\Omega)$, so we deduce that $\lim _{\epsilon \rightarrow 0^{+}} d\left(f_{\epsilon}(x), \Sigma\right)=0$ uniformly. Therefore, there exists $\epsilon_{1} \in\left(0, \epsilon_{0}\right)$ such that

$$
\left|f_{\epsilon}(x)-p+g\right| \geq \frac{r}{2}
$$

for all $x \in \Omega, z \in \mathrm{D}(\mathrm{T}) \cap \bar{\Omega}, \mathrm{g} \in \mathrm{T} z, \epsilon \in\left(0, \epsilon_{1}\right)$.

Thus $\operatorname{deg}\left(f_{\epsilon}+T_{\lambda}, \Omega, p\right)=0$ for all $\lambda \in\left(0, \lambda_{0}(\epsilon)\right)$, and $\epsilon \in\left(0, \epsilon_{1}\right)$. Consequently, it follows from the definition that $\operatorname{deg}(f+T, \Omega \cap D(T), p)=0$, which is a contradiction.

Proposition 2.4. Let $\left\{h_{t}(\cdot)\right\}_{t \in[0,1]}$ be a family of functions in $\mathrm{VMO}(\Omega)$, and $h_{t}(\cdot)$ depends continuously on the parameter $t$ in the topology of $B M O \cap \mathrm{L}_{\text {loc }}^{1}(\Omega)$. Assume that there exists an open neighborhood $\mathrm{U}$ of $\partial \Omega$ in $\Omega$ and a constant $\beta>0$ such that

$$
\frac{1}{m\left(B_{\epsilon}(y)\right)} \int_{B_{\epsilon}(y)}\left|h_{t}(x)+g-p\right| d x \geq \beta
$$

for all $0<\epsilon<\frac{1}{2} d(y, \partial \Omega), g \in T z, z \in D(T) \cap B_{\epsilon}(y), t \in[0,1]$, where $B_{\epsilon}(y)$ is an open ball centered at $y$ with radius $\epsilon$ such that $\overline{B_{\epsilon}(y)} \subset \mathrm{U}$. Then $\operatorname{deg}\left(h_{t}+T, \Omega \cap D(T), p\right)$ does not depend on $t \in[0,1]$.

Proof. Since $h_{t}(\cdot)$ depends continuously on the parameter $t$ in the topology of $\mathrm{BMO}_{\mathrm{O}} \mathrm{L}_{\mathrm{loc}}^{1}(\Omega)$, we have

$$
\lim _{m(B) \rightarrow 0} \frac{1}{m(B)} \int_{B}\left|h_{t}(x)-\overline{h_{t}}\right|=0,
$$

uniformly in t. From (2.5), (2.6), and using the same proof as in (2.3), we know that there exists $\epsilon_{0}>0$, such that

$$
\left|h_{t, \epsilon}(x)+g-p\right| \geq \frac{\beta}{2}
$$

for all $x \in \partial \Omega_{\epsilon} \cap \mathrm{D}(\mathrm{T}), \mathrm{g} \in \mathrm{Tx}, \mathrm{t} \in[0,1], \epsilon \in\left(0, \epsilon_{0}\right)$. By using the same proof as in Lemma 2.2, we know that there exists $\lambda(\epsilon)>0$, such that

$$
p \neq h_{t, \epsilon}(x)+T_{\lambda} x
$$

for all $x \in \partial \Omega_{\epsilon}, t \in[0,1], \lambda \in(0, \lambda(\epsilon))$. Thus $\operatorname{deg}\left(h_{t, \epsilon}+T_{\lambda}, \Omega_{\epsilon}, p\right)$ does not depend on $t$ for each $\epsilon \in\left(0, \epsilon_{0}\right), \lambda \in(0, \lambda(\epsilon))$. Thus $\operatorname{deg}\left(h_{t}+\mathrm{T}, \Omega \cap \mathrm{D}(\mathrm{T}), \mathrm{p}\right)$ does not depend on $t \in[0,1]$.

Corollary 2.5. Let $f_{1}, f_{2} \in \operatorname{VMO}(\Omega)$ satisfying (2.1). Suppose there exists $0<\beta_{0}<\beta$ such that

$$
\frac{1}{m(B)} \int_{B}\left|f_{1}(x)-f_{2}(x)\right| d x<\beta_{0}
$$

for all $\mathrm{B} \subset \mathrm{U}$. Then $\operatorname{deg}\left(f_{1}+\mathrm{T}, \Omega \cap \mathrm{D}(\mathrm{T}), \mathrm{p}\right)=\operatorname{deg}\left(\mathrm{f}_{2}+\mathrm{T}, \Omega \cap \mathrm{D}(\mathrm{T}), \mathrm{p}\right)$.

Proof. Set $h_{t}(x)=t f_{1}(x)+(1-t) f_{2}(x)$ for $t \in[0,1], x \in \Omega$. Then it is easy to see that $h_{t}$ depends continuous on $t$ in the topology of $B M O \cap L_{\text {loc }}^{1}(\Omega)$. Also we have

$$
\frac{1}{m\left(B_{\epsilon}(y)\right)} \int_{B_{\epsilon}(y)}\left|h_{t}(x)+g-p\right| d x \geq \beta-\beta_{0}
$$


for all $0<\epsilon<\frac{1}{2} d(y, \partial \Omega), g \in T z, z \in D(T) \cap B_{\epsilon}(y), t \in[0,1]$, where $B_{\epsilon}(y)$ is an open ball centered at $\mathrm{y}$ with radius $\epsilon$ such that $\overline{\mathrm{B}_{\epsilon}(\mathrm{y})} \subset \mathrm{U}$. Therefore the conclusion follows from Proposition 2.4.

Proposition 2.6. Let $T_{i}: D \subseteq R^{n}, i=1,2$, be two maximal monotone maps. If $t T_{1}+(1-t) T_{2}$ is maximal monotone for each $t \in[0,1]$, and there exist an open neighborhood $\mathrm{U}$ of $\partial \Omega$ in $\Omega$ and a constant $\beta>0$ such that

$$
\frac{1}{m\left(B_{\epsilon}(y)\right)} \int_{B_{\epsilon}(y)}\left|f(x)+g_{t}-p\right| d x \geq \beta
$$

for all $\left.0<\epsilon<\frac{1}{2} \mathrm{~d}(\mathrm{y}, \partial \Omega), \mathrm{g}_{\mathrm{t}} \in\left[\mathrm{tT} \mathrm{T}_{1}+(1-\mathrm{t}) \mathrm{T}_{2}\right] z, z \in \mathrm{D} \cap \mathrm{B}_{\epsilon}(\mathrm{y})\right), \mathrm{t} \in[0,1]$, where $\mathrm{B}_{\epsilon}(\mathrm{y})$ is an open ball centered at $y$ with radius $\epsilon$ such that $\overline{B_{\epsilon}(y)} \subset \mathrm{U}$. Then $\operatorname{deg}\left(f+\left[\mathrm{tT}_{1}+(1-t) T_{2}\right], \Omega \cap \mathrm{D}, \mathrm{p}\right)$ does not depend on $t \in[0,1]$.

Proof. By (2.8), using the same proof as in (2.3), we know that there exists $\epsilon_{0}>0$, such that

$$
\left|f_{\epsilon}(x)+g_{t}-p\right| \geq \frac{\beta}{2},
$$

for all $\left.x \in \partial \Omega_{\epsilon} \cap \mathrm{D}, \mathrm{g}_{\mathrm{t}} \in \mathrm{tT}_{1} x+1-\mathrm{t}\right) \mathrm{T}_{2} x, \mathrm{t} \in[0,1], \epsilon \in\left(0, \epsilon_{0}\right)$. From (2.9), and using the same proof as in Lemma 2.2, we know that there exists $\lambda(\epsilon)>0$, such that

$$
p \neq f_{\epsilon}(x)+T_{\lambda}^{t} x
$$

for all $x \in \partial \Omega_{\epsilon}, t \in[0,1], \lambda \in(0, \lambda(\epsilon))$, where $T_{\lambda}^{t}$ is the Yosida approximation of $t T_{1}+(1-t) T_{2}$. From Lemma 2.7 in [15], we know

$$
\operatorname{deg}\left(f_{\epsilon}+T_{\lambda}^{t}, \Omega_{\epsilon}, p\right)
$$

does not depend on $t \in[0,1], \lambda \in(0, \lambda(\epsilon))$. Therefore, $\operatorname{deg}\left(f+\left[t T_{1}+(1-t) T_{2}\right], \Omega \cap D, p\right)$ does not depend on $t \in[0,1]$.

Acknowledgement: The first author was supported by a NSFC grant, grant no. 10871052 .

Received: April 2009. Revised: May 2010.

\section{Referencias}

[1] A. Boutet de Monvel-Berthier, V. Georgescu and R. Purice, A boundary value problem related to the Ginzburg-Landau model, Comm. Math. Phys 1991 pag. 1-23.

[2] J. Bourgain, H. Brezis and P. Mironescu, Lifting in Sobolev spaces, J. Analyse Math. 2000 pag. $37-86$.

[3] J. Bourgain, H. Brezis and P. Mironescu, Another look at Sobolev spaces, in Optimal Control and Partial Differential Equations, (J.L. Menaldi, E. Rofman et A. Sulem, eds), a volume in honour of A. Bensoussanj ${ }^{-}$s 60 th birthday IOS Press 2001 pag. 439-455. 
[4] J. Bourgain, H. Brezis and P. Mironescu, H1/2 maps into the circle: minimal connections, lifting, and the Ginzburg-Landau equation, Publications mathjäematiques de $\mathrm{l}_{i}^{-}$IHES 2004 pag. $1-115$.

[5] J. Bourgain, H. Brezis and P. Mironescu, Lifting, Degree and Distributional Jacobian Revisited, Comm. Pure Appl. Math. 2005 pag. 529-551.

[6] J. Bourgain, H. Brezis and H.-M. Nguyen, A new estimate for the topological degree, C. R. Acad. Sc. Paris 2005 pag. 787-791.

[7] H. Brezis, Operateurs Maximaux monotones North-Holland 1973.

[8] H. Brezis And J. M. Coron, Large solutions for harmonic maps in two dimensions, Comm. Math. Phys. 1983 pag. 203-215.

[9] H. Brezis, Degree theory: old and new, in Topological Nonlinear Analysis II: Degree, Singularity and Variations, (M. Matzeu and A. Vignoli ed.) Birkhauser 1997 pag. 87-108.

[10] H. Brezis And Y. Li, Topology and Sobolev spaces, J. Funct. Anal. 2001 pag. 321-369.

[11] H. Brezis, Y. Li, P. Mironescu and L. Nirenberg, Degree and Sobolev spaces Topological methods in Nonlinear Analysis 1999 pag. 181-190.

[12] H. Brezis and L. Nirenberg, Degree theory and BMO, Part I : compact manifolds without boundaries Selecta Math. 1995 pag. 197-263.

[13] H. Brezis and L. Nirenberg , Degree Theory and BMO, Part II: Compact Manifolds with Boundaries Selecta Math. 1996 pag. 1-60.

[14] D. O'Regan, Y. J. Cho, Y. Q. Chen, Topological Degree Theory and Applications. Chapman and Hall/CRC Press 2006.

[15] Y.Q.Chen, D.O'Regan, On the homotopy property of topological degree for maximal monotone mappings. Appl. Math. Comput. 2009 pag. 373-377.

[16] Y.Q.Chen, D. O'Regan, F.L.Wang, R. Agarwal A note on degree theory for maximal monotone mappings in finite dimensional spaces, Appl. Math. Lett. 2009 pag. 1766-1769.

[17] M.J. Esteban And S. Milller, Sobolev maps with integer degree and applications to Skyrme's problem, Proc. Roy. Soc. London A 1992 pag. 197-201.

[18] M. Giaquinta, G. Modica And J. Soucek, Remarks on the degree theory, J. Funct. Anal. 1994 pag. 172-200.

[19] F.B. Hang and F.H. Lin, Topology of Sobolev mappings II, Acta Math. 2003 pag. 55-107.

[20] F. John and L. Nirenberg, On functions of bounded mean oscillation, Comm. Pure Appl. Math. 1961 pag. 415-426. 
[21] J. KorevaAR, On a question of Brezis and Nirenberg concerning the degree of circle maps Selecta Math. 1999 pag. 107-122.

[22] P. Mironescu And A. Pisante, A variational problem with lack of compactness for H1/2(S1, S1) maps of prescribed degree. J. Funct. Anal. 2004 pag. 249-279.

[23] D. Sarason, Functions of vanishing mean oscillation Trans. Amer. Math. Soc. 1975 pag. 391-405 